\title{
Gas-driven hydrogen isotopes permeation through different carbon materials
}

\author{
A.V. Spitsyn ${ }^{\mathrm{a}}$, A.V. Golubeva ${ }^{\mathrm{a}^{*}}$, M. Mayer ${ }^{\mathrm{b}}$, A.A. Skovoroda ${ }^{\mathrm{a}}$ \\ ${ }^{a}$ RRC 'Kurchatov Institute', Moscow, Russia \\ ${ }^{b}$ Max-Planck-Institut für Plasmaphysik, EURATOM Association, Garching, Germany
}

\begin{abstract}
Hydrogen gas driven permeation through the fine-grain graphites MPG-8 and R5710, and through CFC NB31 in two main directions has been investigated for sample thicknesses of $1-7 \mathrm{~mm}$ and pressures from $10^{-2}-10 \mathrm{~Pa}$. The gas driven permeation occurs through the carbon-base materials by the hydrogen molecular gas flow through the internal porosity network rather than hydrogen atom diffusion through the graphite lattice. The permeabilities of MPG-8 and Nb31 are of the same order, while the permeability of R5710 is two orders of magnitude less. The specific bulk conductivity is about $5 \times 10^{15}$ molecules $\cdot \mathrm{s}^{-1} \mathrm{~m}^{-1} \mathrm{~Pa}^{-1}$ for MPG$8 ; 8 \times 10^{13}$ molecules $\cdot \mathrm{s}^{-1} \mathrm{~m}^{-1} \mathrm{~Pa}^{-1}$ for $\mathrm{R} 5710 ; 1 \times 10^{16}$ molecules $\cdot \mathrm{s}^{-1} \mathrm{~m}^{-1} \mathrm{~Pa}^{-1}$ for $\mathrm{Nb} 31$ along expitch $(x)$ fibers after taking into account the perforated surface layer and $0.8 \times 10^{16}$ molecules $\cdot \mathrm{s}^{-}$ ${ }^{1} \mathrm{~m}^{-1} \mathrm{~Pa}^{-1}$ for $\mathrm{Nb} 31$ along needled $(z)$ fibers.
\end{abstract}

PACS: 52.40.Hf, 66.30.-h

PSI-18 keywords: Carbon-based materials, Divertor material, Permeation, Recycling JNM keywords: graphite, divertor materials, permeation

Corresponding author address: Ac. Kurchatov sq., 1/1, Moscow, RU-123182, Russia

Corresponding author e-mail: anna-golubeva@yandex.ru

Presenting author: Dr. Alexander Spitsyn

Presenting author e-mail: spitsyn@nfi.kiae.ru 


\section{Introduction}

Carbon-based materials, such as fine-grain graphites and carbon fiber composites (CFCs) are used in fusion research devices as plasma-facing components (PFCs). CFC's are also intended to be used as PFC in ITER. The accumulation of tritium is one of the key problems for ITER and future thermonuclear reactors: For safety reasons its inventory in PFCs must be strongly limited, and leakages (for example to the coolant) must be minimized. Tritium may be accumulated by co-deposition with eroded carbon or beryllium. A deep diffusion of hydrogen into CFC was observed in laboratory experiments [1], and this deep diffusion and trapping inside the material might be responsible for the high $\mathrm{D}$ inventory observed in Tore Supra [2], where large areas of CFC material are exposed to the plasma at the CIEL limiter. The mechanism of deep tritium permeation in carbon-based materials is thought to be migration through open porosity. The porous structure strongly influences the hydrogen gas permeation through PFCs.

Interaction of hydrogen isotopes with graphites and carbon based materials has been actively investigated during the last decades. At the same time, only few publications were devoted to hydrogen permeation through isotropic graphites $[3,4,5,6]$ and not much is known about the permeability of CFCs.

This work is devoted to experimental investigations of the gas driven permeation (GDP) through carbon-based materials.

\section{Experimental}

\subsection{Materials}

Three materials were investigated: fine-grain graphites MPG-8 and R5710, and carbon fiber composite $\mathrm{Nb} 31$.

The fine-grain graphite MPG-8 was developed in Russia for thermonuclear applications and is used in most Russian tokamaks. It contains a relatively low amount of 
impurities: $\mathrm{Si}<3 \cdot 10^{-5} \%, \mathrm{Fe}, \mathrm{Mn}, \mathrm{Mg}, \mathrm{Al}, \mathrm{Cu}, \mathrm{Ti}, \mathrm{B}<10^{-5} \%$ [7]. Its density is $1.61 \mathrm{~g} / \mathrm{cm}^{3}$, average grain size is $8 \mu \mathrm{m}$, porosity 23 vol. \% (from which 17 vol. $\%$ is open porosity).

R5710 is an ultra-pure fine-grain graphite initially developed for fission applications. It is used for example in ASDEX Upgrade. Its density is $1.88 \mathrm{~g} / \mathrm{cm}^{3}$, average grain size $3 \mu \mathrm{m}$, open porosity 10 vol. $\%$, medium pore size $0.6 \mu \mathrm{m}$. The impurities contents are less than $100 \mathrm{ppm}$.

NB31 is a carbon fiber composite material, and initially foreseen as reference material for ITER. It is a three-directional CFC material, consisting of ex-pitch $(x)$, ex-PAN $(y)$ and needled fibers ( $z$, ex-PAN) [8]. The density of the material is $\rho=1.87-1.94 \mathrm{~g} / \mathrm{cm}^{3}$, the open porosity is $7-9 \%$ [9]. As $\mathrm{Nb31}$ is anisotropic, samples with different orientations were manufactured: along ex-pitch fibers (Nb31-x), and along needled fibers (Nb31-z). The photos of the samples (Fig. 4) and their surface profiles (Fig. 3) show more pronounced deep gaps at the surfaces of ex-pitch samples.

The Nb31 and R5710 samples were manufactured by spark erosion. The MPG-8 samples were mechanically cut without lubricant. No additional samples treatment was performed, except for the cases specially mentioned below. All samples had the shape of disks $\varnothing 27 \mathrm{~mm}$. The thicknesses of the samples varied: for MPG- 8 from 1 to $4.38 \mathrm{~mm}$, for $\mathrm{R} 5710$ from 1 to $2.5 \mathrm{~mm}$, for Nb31- $x$ from 2 to $7 \mathrm{~mm}$, for $\mathrm{Nb} 31-z$ from 1 to $5.5 \mathrm{~mm}$.

\subsection{Set-up}

The experimental investigation of the permeability was performed at the installation described in [6], but modified for the presented experiments. A scheme of the experimental unit is shown in Fig. 1.

The sample separates two vacuum volumes (A and B) and is vacuum-sealed with two gaskets of vacuum rubber. The surface exposed to the gas loading is constrained by limiters 
and has $\varnothing 20 \mathrm{~mm}$. The side face of the sample is surrounded with a shield, used both for sample centering and suppressing gas leakages through the edge.

In the experiments the hydrogen gas flux through the membrane was measured with a pressure gradient at the inlet and outlet surfaces. In the inlet chamber A a hydrogen pressure in the range of $10^{-2}-10 \mathrm{~Pa}$ was established. Hydrogen flux permeating through the sample comes into the measuring chamber $\mathrm{B}$, which has no own pumping. The permeating gas is pumped out from chamber B through a calibrated orifice between chambers B and C. Gas pressures in vacuum chambers $\mathrm{A}, \mathrm{B}$ and $\mathrm{C}$ are controlled by high-pressure ionization gauges with filaments covered by yttrium oxide. The gas flux Q though the orifice is determined from the pressures before and after the diaphragm:

$$
\mathrm{Q}\left[\mathrm{H}_{2} / \mathrm{sec}\right]=\mathrm{s} \cdot\left(\mathrm{P}_{\mathrm{B}}-\mathrm{P}_{\mathrm{C}}\right)
$$

where $\mathrm{s}$ - orifice gas conductivity, $\mathrm{s}=0.741 \cdot \mathrm{s}^{-1} ., \mathrm{P}_{\mathrm{B}, \mathrm{C}}$ - pressures measured before and after the diaphragm.

\section{Results and discussion}

For all materials investigated the hydrogen permeating flux increases proportional to the pressure gradient at the membrane sides in the range of $10^{-2} \div 80 \mathrm{~Pa}$ (Fehler! Verweisquelle konnte nicht gefunden werden.), and is inversely proportional to the sample thickness. A set of additional experiments with argon GDP was performed and has demonstrated for all used materials that the permeating flux is inversely proportional to the square root of the molecular mass.

Though being totally different types of materials (fine-grain graphite and CFC), the permeating fluxes through MPG-8 and Nb31 are of the same order, while the permeating flux

through the fine-grain graphite R5710 is smaller by two orders of magnitude (Fehler! Verweisquelle konnte nicht gefunden werden.). The permeability of $\mathrm{Nb} 31$ depends on the direction (Fehler! Verweisquelle konnte nicht gefunden werden.). 


\subsection{Specific gas conductivity}

The experimentally observed permeation cannot be described by atomic hydrogen diffusion through the graphite lattice. Diffusion limited atomic permeation is characterized by a square root dependence of the permeation rate on the loading pressure, while we observe a linear dependence. The permeation of hydrogen through the samples of porous carbon materials (MPG-8, R5710 and Nb31) can be formally described as the gas flow in the molecular mode through curvilinear channels formed by the interconnected opened porosity in the bulk.

The gas flow in a capillary is inversely proportional to the capillary length, inversely proportional to the square root of the molecular mass and proportional to pressure loading in the molecular regime of gas flow [10]. All these peculiarities were observed in our experiments.

From the obtained pressure and thickness dependences one can write the permeation flux through a carbon (graphite or CFC) membrane as

$$
\mathrm{j}=\sigma \cdot \Delta P \cdot A / d,
$$

where $\sigma$ is the specific gas conductivity of the membrane, $\Delta P$ is the pressure difference, $A$ is the surface area and $d$ the thickness of the membrane. The $\sigma$ here is the characteristics of the material rather than the properties of the sample (thickness, area). The $\sigma$ data obtained in the present work for the three carbon-based materials under investigation are summarized in Table 1.

\subsection{Structure dependence of permeability}

The observed difference of the permeating fluxes by two orders of magnitude between R5710 and MPG-8 fine-grain graphites has the only explanation in a different porosity and different typical void size. The open porosity is 10 vol. \% for R5710 and 17 vol. \% for MPG-

8. The mean grain sizes of R5710 and for MPG- 8 are $3 \mu \mathrm{m}$ and $8 \mu \mathrm{m}$, respectively. The void 
size should follow the grain sizes. The dependence of the permeating flux on the typical void size is strongly nonlinear. The same situation was previously reported in [11], where an increase of the open porosity of MPG- 8 from 17 vol. \% to 19 vol. \% resulted in an increase of the permeating flux by a factor of two. Such a strong influence of the structure on gas permeability of carbon-based materials should be taken into account when selecting materials for fusion reactors.

\subsection{Scatter of specific conductivity data}

For the fine-grain graphites the scatter of the data for different samples of the same thickness is less than $30 \%$ from the mean value.

For Nb31 the permeability measurements for the same sample are repeatable, while for different samples with the same orientation the $\sigma$ data scatter more strongly (Table 1): $60 \%$ from the mean value for $\mathrm{Nb} 31-x$ and 2-3 times for $\mathrm{Nb} 31-z$.

A large scatter of the data for $\mathrm{Nb31-z}$ is explained by the pronounced laminated structure of $\mathrm{Nb} 31$ in this direction. Fig. 4.a shows sample cross-sections $(y ; z)$ : along the axis $z$ layers of carbon fibers and pitch take turns with a period of $1.4 \mathrm{~mm}$. A $1 \mathrm{~mm}$ thick sample therefore can consist mainly of fiber or of pitch, and the permeating flux for these two cases should be different. This effect should decrease with increasing sample thickness, so the results for the thicker samples $\left(0.8 \times 10^{16}\right.$ molecules $\cdot \mathrm{s}^{-1} \mathrm{~m}^{-1} \mathrm{~Pa}^{-1}$ for Nb31-z) are more reliable in representing mean material properties.

For Nb31- $x$ a decrease of $\sigma$ with increasing sample thickness was observed (

Fig. 5). The material is homogeneous in $x$ direction, and the only reason for $\sigma$ scatter is a different roughness of samples. For Nb31-x the surface relief is very pronounced in comparison with Nb31-z, as can be seen from profilometry data (Fig. 3). The origin of gaps is likely the detachment of the matrix when cutting the material. 
The gas conductivity of a surface layer with multiple deep gaps is much higher than that of a smooth surface. To obtain results characterizing the bulk properties of the material it is necessary to subtract the typical thickness $h$ of the perforated layer from the membrane thickness when calculating $\sigma$. Then the calculation should give the same results for samples of different thicknesses. From this we obtained $h$ to be equal $0.9 \mathrm{~mm}$. Correction of the $\sigma$ calculations gave the same value of $1 \times 10^{16}$ molecules $\cdot \mathrm{s}^{-1} \mathrm{~m}^{-1} \mathrm{~Pa}^{-1}$ for all samples except the thinnest one ( $2 \mathrm{~mm}$ thick).

\section{Conclusion}

The hydrogen gas driven permeation through membranes of different carbon-based materials: fine grain graphites MPG-8 and R5710, and CFC NB31 in directions $x$ and $z$ was investigated.

The mechanism of permeation through carbon-based materials is hydrogen gas flow through the interconnected porosity and channels in the molecular regime. For all investigated materials the specific gas conductivity $\sigma$ was obtained, which can be used for characterization of the bulk material.

The permeability of carbon-based materials strongly depends on its structure. For two fine-grain graphites investigated with a grain size of 3 and $8 \mu \mathrm{m}$ the permeability differs by two orders of magnitude.

The gas permeability of carbon materials probably explains the observed deep diffusion and deep trapping of deuterium in porous graphite materials [1]: Some hydrocarbon radicals and hydrogen molecules can penetrate deep into the material along the open porosity and then adsorb at the walls of the pore. 


\section{Acknowledgements}

The authors are thankful to V. Svischev for his help in experiments and to S. Kamneva for samples surface profile measurement.

This work is supported by the Impuls und Vernetzungsfond of the Helmholtzgemeinschaft, the joint Russian Foundation for Basic Researches - HelmholtzGemeinschaft grant 07-08-92280, Russian Foundation for Basic Researches grant 05-0850023, the International Science and Technological Center (Grant 2805), RRC Kurchatov Institute grant program for junior researches, Russian Program for Leading Scientific School (371.2008.2). 
Table 1 . Specific gas conductivity $\sigma$

\begin{tabular}{|l|l|l|}
\hline Material & $\begin{array}{l}\text { sample } \\
\text { thickness, } \\
\text { mm }\end{array}$ & $\begin{array}{l}\sigma, 10^{16} \\
\text { molecules } \cdot \mathrm{s}^{-1} \mathrm{~m}^{-1} \mathrm{~Pa}^{-1}\end{array}$ \\
\hline MPG-8 & $1.65^{(*)}$ & $0.503 \pm 0.002$ \\
\hline R5710 & $1-2.5$ & $0.008 \pm 0.002$ \\
\hline Nb31-x & 2.0 & 4,1 \\
& 2.5 & 3,7 \\
& $2.5^{(*)}$ & $3.7 ; 3.6$ \\
& 3.0 & $2.8 ; 2.4 ; 2.8 ; 3.0$ \\
& $7.0^{(*)}$ & $1.35 ; 1.3$ \\
\hline Nb31-z & 1.0 & $3.2 ; 1.8$ \\
& 1.5 & 1.08 \\
& 2.0 & 1.2 \\
& 2.5 & $0.48 ; 1.04$ \\
& $2.5^{(*)}$ & $0.5 ; 0,5$ \\
& 3.0 & $0.81 ; 1.15 ; 1.05$ \\
& 5.5 & 0.81 \\
\hline
\end{tabular}

*Two values of $\sigma$ in one line means two independent measurements with the same sample 


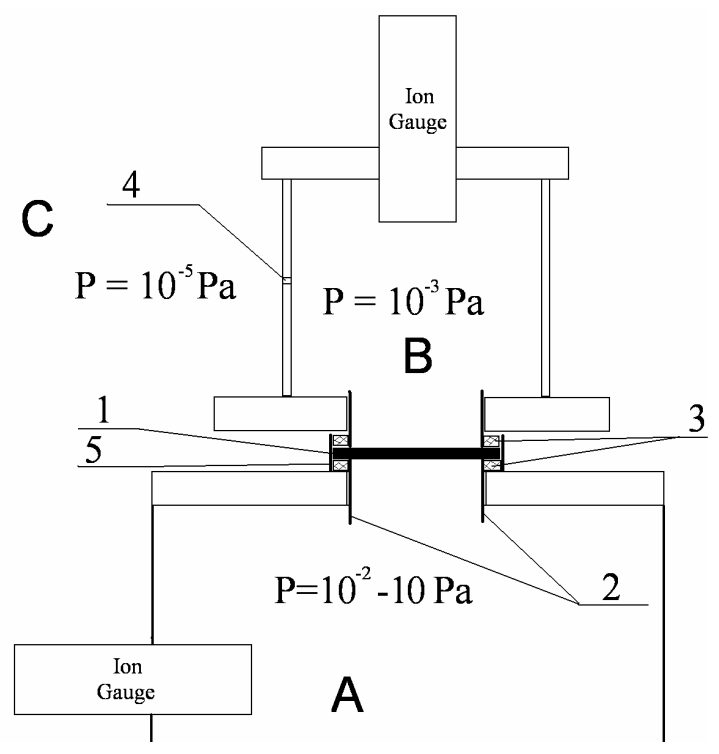

Fig. 1. Sample holder. 1 - sample; 2 - rubber limiter; 3 - rubber; 4 - measuring orifice; 5 limiter \& centering ring. 

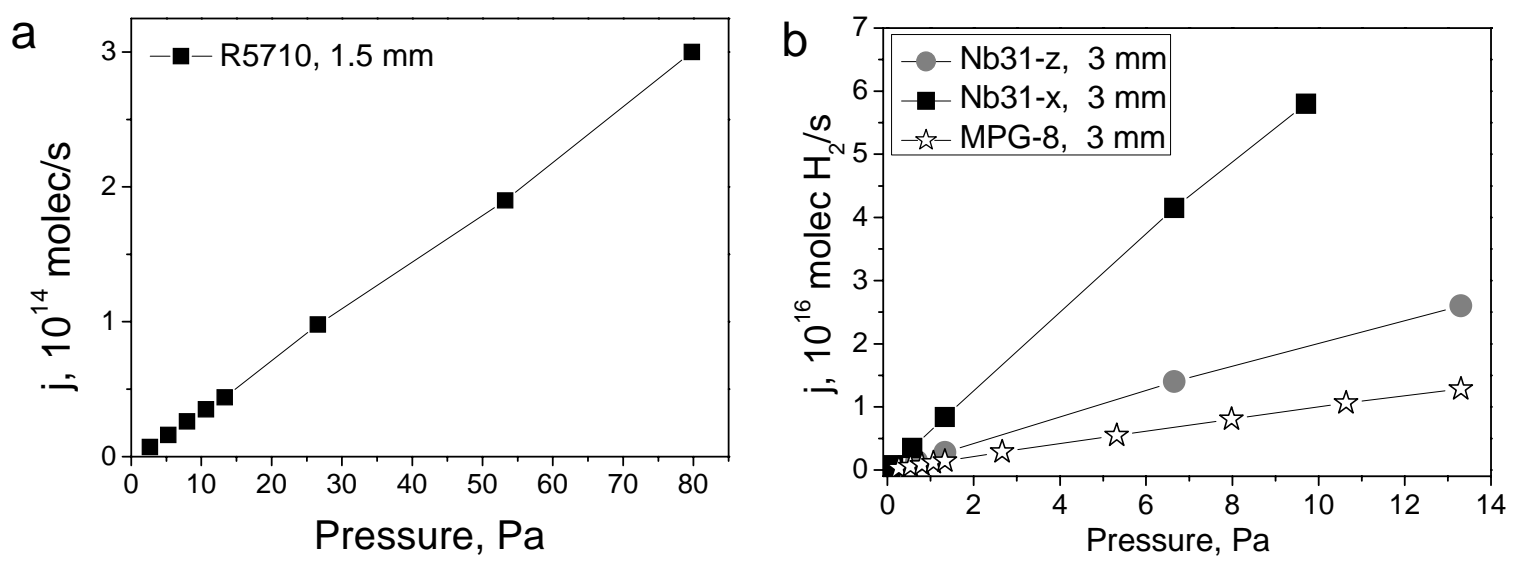

Fig. 2. Permeation flux through graphite materials as a function of gas pressure:

$\mathrm{a}$ - fine grain graphite R5710, b - MPG-8, Nb31-x and Nb31-z samples. Area of the samples$3,15 \mathrm{~cm}^{2}$. 


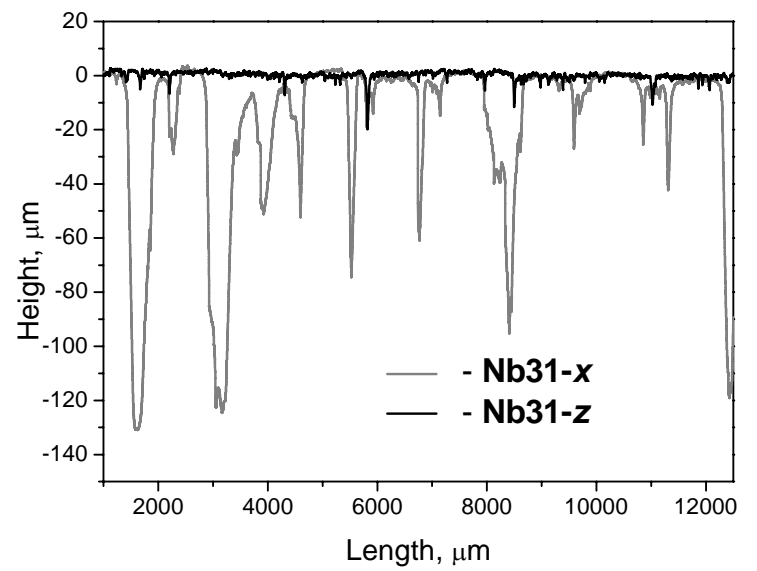

Fig. 3. Surface profiles of Nb31-x and Nb31-z samples. 

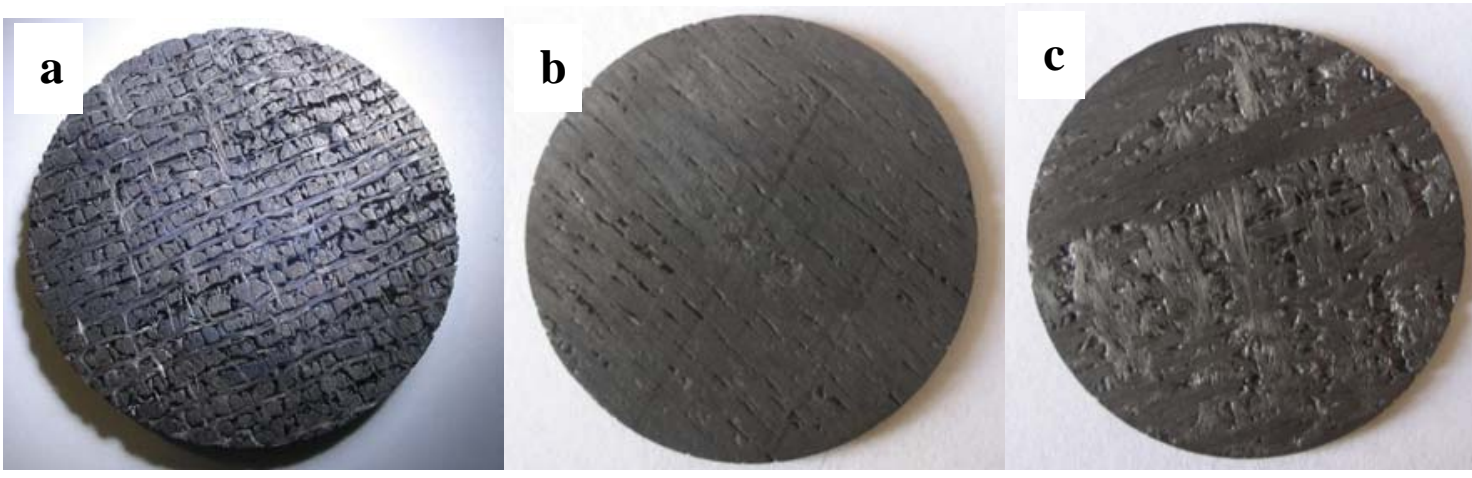

Fig. 4. Photos of Nb31 samples $\varnothing 27 \mathrm{~mm}: \mathrm{a}-\mathrm{Nb} 31-x, \mathrm{~b}-\mathrm{Nb} 31-z$, cross section cut through fiber layer, $\mathrm{c}-\mathrm{Nb31-z}$ cross section cut through pitch layer. Samples diameter 27 $\mathrm{mm}$. 


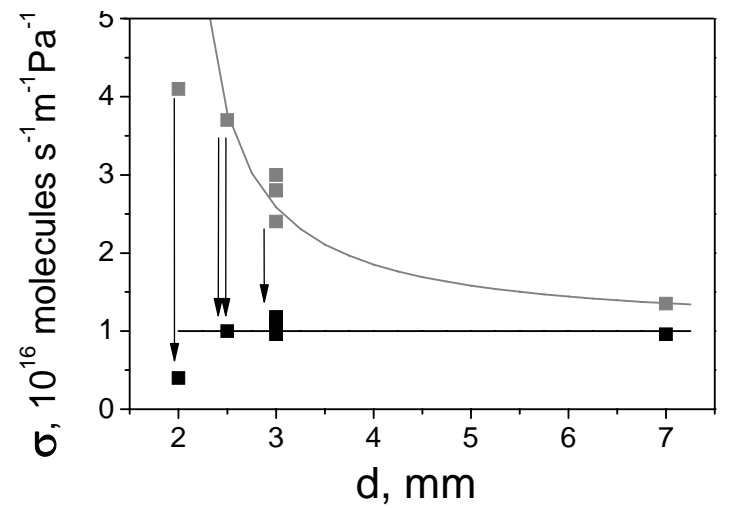

Fig. 5. The specific $\sigma$ conductivity of Nb31-z: $\square$ - experiment, $\mathbf{a}$ — after correction taking into account surface gappy layer $(0,9 \mathrm{~mm})$ 


\section{References}

1. J. Roth, V.Kh. Alimov, A.V. Golubeva, R.P. Doerner, J. Hanna, E. Tsitrone, Ch. Brosset, V. Rohde, A. Herrmann, M. Mayer, J. Nucl. Mater. 363-365 (2007) 822.

2. E. Tsitrone, J. Nucl. Mater. 363-365 (2007) 12.

3. T.Kiyoshi, T.Namba, M.Yamawaki, J.Nucl.Mater, 155-158 (1988) 230-233.

4. M.Yamawaki, K.Yamaguchi, Y.Suzuki, S.Tanaka, J.Nucl.Mater. 179-181 (1991) 239-241.

5. K.Yamaguchi, Y.Suzuki, S.Tanaka, M.Yamawaki, J.Nucl.Mater. 179-181 (1991) 242-245.

6. A. Spitsyn, A. Pisarev, A. Skovoroda, V. Gureev, Yu. Martynenko. J.Nucl.Mater. 363-365 (2007) 833-838.

7. Dobrokhotov E.I., Dremin M.M., Pavlov Yu.D., Petrov D.P., Solntsev A.M.,

Stefanovskij A.M., Terebkov A.L., Shcherbak A.F. — 9th International Conference on

Plasma Physics and Controlled Fusion, Baltimore, 1982, v.III, p.229.

8. A T Peacock, M. Merola, M. A. Pick, R. Tivey, Phys. Scr. T128 (2007) 23-28.

9. A. Kreter, S. Brezinsek, M. Rubel, B. Emmoth, M. Freisinger, P. Pelicon, V. Philipps, O.

Schmitz, P. Sundelin, G. Sergienko and TEXTOR team, J. of Physics: Conference Series 100 (2008) 062024.

10. "Design and construction of vacuum system", Pipko I.A., Moscow, "Energy", 1978.

11. V.S. Petrov, A.A. Polunina, M.V. Provotorov, A.A. Skovoroda, A.V.

Spitsyn, T.N. Trushkova // Voprosy Atomnoi Nauki i Tehniki, ser.:

Termoyadernyi Sintez (Topics in Atomic Science and Technology, ser.:

Termonuclear fusion, in Russian) - 3 (2008) 61-67 